This is a post-peer-review, pre-copyedit version of a conference paper published in Microactuators and Micromechanisms. Mechanisms and Machine Science 45 : 153-164 (2017). The final authenticated version is available online at: http://dx.doi.org//10.1007/978-3-319-45387-3_14

\title{
Dynamic model of a compliant 3PRS parallel mechanism for micromilling
}

\author{
A. Ruiz ${ }^{1}$, F.J. Campa ${ }^{1}$, C. Roldán-Paraponiaris ${ }^{1}$, O. Altuzarra ${ }^{1}$ \\ ${ }^{1}$ Department of Mechanical Engineering, Compmech Research Group, University of the Basque \\ Country UPV/EHU, Spain; e-mail: antonio.ruiz@ehu.es; fran.campa@ehu.es (corresponding \\ author); constantino.roldan@ehu.es; oscar.altuzarra@ehu.es
}

\begin{abstract}
The final objective of this work is to reach a manipulator of 5 degrees of freedom which is capable of developing micromilling operations. It consists of a XY stage under a 3PRS compliant parallel mechanism, obtaining the advantages of the compliant joints as are higher repetitiveness, smoother motion and a higher bandwidth, due to the high precision demanded from the process, under 0.1 micrometers. In this work, the dynamics of the compliant stage will be developed. The modelling approach is based on the use of the Principle of Energy Equivalence combined with the Boltzmann-Hamel equations to analyze the rotational dynamics of the platform. A pseudo-rigid model has been assumed for the compliant joints, calculating the flexural and torsional stiffness by FEA. Finally, a prototype has been built and some preliminary results are shown comparing the simulation and the measurements.
\end{abstract}

Keywords Compliant mechanism, Parallel mechanism, Dynamics

\section{Introduction}

Nowadays, micromilling applications have and a great demand in different areas as scientific, medical, metrological and communication fields. The requirements for that type of operations are very restrictive in terms of precision and surface finish. The employed machines used in this sector are too large compared to the demanded workspace for several applications. For that reason, the design of smaller and more compact machines is desirable, which is the objective of the present work.

Here, the development of a compact micromilling machine based on a compliant parallel manipulator is proposed. The workpiece will be supported on a 3PRS compliant parallel mechanism (Yue et al. 2010) in series with an XY stage whereas the high speed spindle will remain on a fixed gantry. The 3PRS solution is a fusion of a compliant mechanism (Howell 2001) and a parallel mechanism (Merlet 2000), so the advantages of both devices can be obtained: no friction, not need lubrication and zero backlash from the flexure devices and the higher mechanical stiffness, higher loading capacity, and higher positioning accuracy from the parallel mechanism. 
The use of compliant mechanisms in high precision applications is becoming very common. For example, in (Kim and Choi 2012), a mechanism for a single-axis flexure-based nano-positioning stage (1dof) with a range of motion up to a millimeter and a compact stage size is presented. For mechanisms with 2 dof, some different configurations have been developed. A planar motion stage design based on flexure elements is showed in (Wang et al. 2011). Another example of this type of device is resented in (Yong et al. 2009), where a device with a relatively large range and high scanning speed is showed. Also, 3 dof mechanisms have been proposed. For example, an ultra-precision $\mathrm{XY} \theta \mathrm{z}$ flexure stage with nanometer accuracy is presented by Kim et al. (Kim et al. 2010). A high-performance threeaxis serial-kinematic nano-positioning stage for high-bandwidth applications is developed by Kenton and Leang (Kenton and Leang 2012). Other design of a XYZ Compliant Parallel Manipulator is showed in (Hao and Kong 2012), where the structure is composed by identical spatial double four-beam modules.

On the other hand, the manufacturing of microlenses consists of milling a matrix of NxN concave aspherical cavities with the negative of the lens on one of a cylindrical workpiece, which diameter ranges from 10 to $20 \mathrm{~mm}$, see Fig. 1. Different shapes of the lens can be developed, ranging diameters from $0.5 \mathrm{~mm}$ to $2 \mathrm{~mm}$ and sagittal depths less than $1 \mathrm{~mm}$. The kinematic requirements in terms of needed displacements have been obtained by means of the design of a standard process, where a matrix of $4 \times 4$ cavities in a cylindrical mould of $18 \mathrm{~mm}$ is achieved. The cavities are spherical with a diameter of $2 \mathrm{~mm}$, and are machined in downmilling with a spiral down strategy in counter-clock direction. The offset between the part and the tool tip for the motions between cavities is of $1 \mathrm{~mm}$. The resulting displacements needed in the workpiece are $\pm 10 \mathrm{~mm}$ in $\mathrm{X}$ and $\mathrm{Y}$.
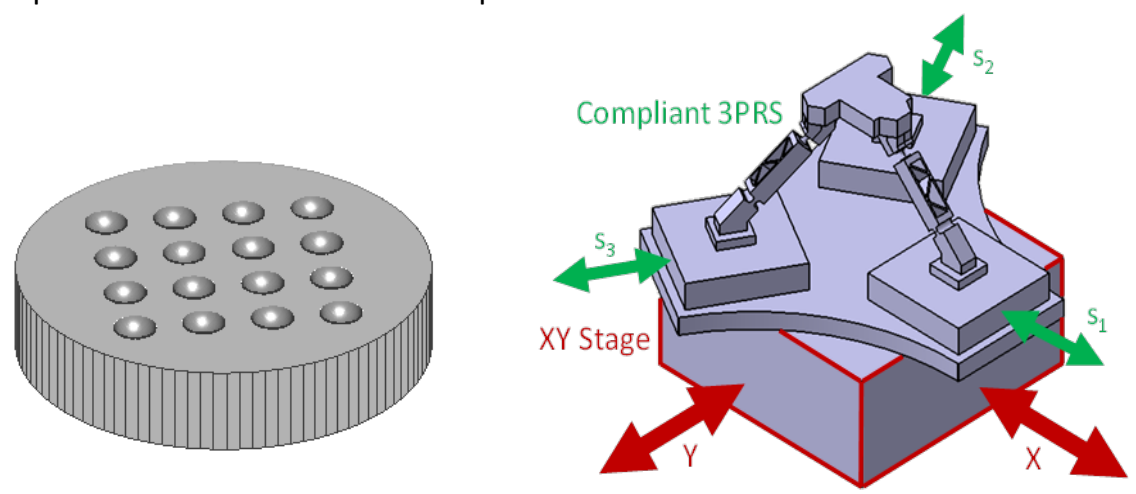

Fig. 1 Left) CAD model of a microlenses mould. Right) Schematic of a XY3PRS manipulator

To perform this operation, a XY3PRS hybrid mechanism has been selected. It is based on a 3PRS compliant parallel kinematics manipulator mounted in series on a $X Y$ stage, see Fig. 1. The moving platform is connected to the $X Y$ stage by each leg, where a $\mathrm{P}$ joint, a $\mathrm{R}$ joint and a $\mathrm{S}$ joint are placed in sequence and the $\mathrm{P}$ joint is actuated by a linear actuator. Thus, three identical PRS linkages attach the moving 
platform to the base ( $\mathrm{Li}$ and $\mathrm{Xu}$ 2007). This configuration allows meeting the requirements of the process and offers the option of performing rotations around the $X$ and $Y$ axis. this offers the possibility of performing rotations around the $X$ and $Y$ axis, which would allow a better orientation of the ball end mill with respect to the workpiece.

The present study is focused on the dynamic analysis of the 3PRS compliant manipulator, whose rigid body kinematics has been previously solved following the work from ( $\mathrm{Li}$ and Xu 2007). That dynamic model will be used for cosimulation of the mechatronics of the manipulator and the milling process.

\section{Dynamic Analysis}

The dynamic problem has been solved using the Principle of Energy Equivalence, that is, the mechanism will be divided in $b$ open-chain subsystems where the Lagrange equations can be applied with their local generalized coordinates $q_{b}$. The condition that the $b$ individual subsystems move like belonging to an assembled mechanism implies that $q_{B}$, the set of all the generalized coordinates $q_{b}$ is a function of the generalized coordinates $q$ of the assembled mechanism. Thus, the virtual displacements can be related by means of the Jacobian. Furthermore, as the movement is the same, the virtual work performed by the assembly system and by the set of subsystems must be the same:

$$
\delta W=\delta W_{B} \Rightarrow \boldsymbol{\delta} \mathbf{q}^{\mathbf{T}} \boldsymbol{\tau}=\boldsymbol{\delta} \mathbf{q}_{\mathbf{B}}^{\mathbf{T}} \boldsymbol{\tau}_{\mathbf{B}}=\boldsymbol{\delta} \mathbf{q}^{\mathbf{T}} \mathbf{J}^{\mathbf{T}} \boldsymbol{\tau}_{\mathbf{B}}
$$

Hence, the actuating forces on the assembled mechanism can be calculated as:

$$
\boldsymbol{\tau}=\mathbf{J}^{\mathbf{T}} \boldsymbol{\tau}_{\mathbf{B}}=\sum_{b=1}^{B} \mathbf{J}_{\mathbf{b}}^{\mathbf{T}} \boldsymbol{\tau}_{\mathbf{B}}
$$

This method was covered in (Altuzarra et al. 2015) and will be applied to the compliant 3PRS in the following sections.

\subsection{Compliant 3PRS description and hypotheses}

The schematics of the compliant 3PRS is shown in Fig. 2. The length of the legs is $L$, and the radius from the center of the moving platform to the center of the spherical joints is $b$. Three prismatic actuators at 120 o have been employed, being the joint space coordinates $s_{1}, s_{2}$, and $s_{3}$, which indicate the position of the center 
of the revolute joints. Those will be the generalized coordinates of the 3PRS mechanism. The angles between the legs and the XY stage are $\alpha_{i}$, whose values are 45은 in the default position. As a result, the platform is able to perform two rotations around $X$ and $Y$ axis, $\psi$ and $\theta$, respectively, as well as movements in $Z$ direction. Parasitic motions in $\mathrm{X}, \mathrm{Y}$ and a rotation around $\mathrm{Z}, \phi$, also appear.

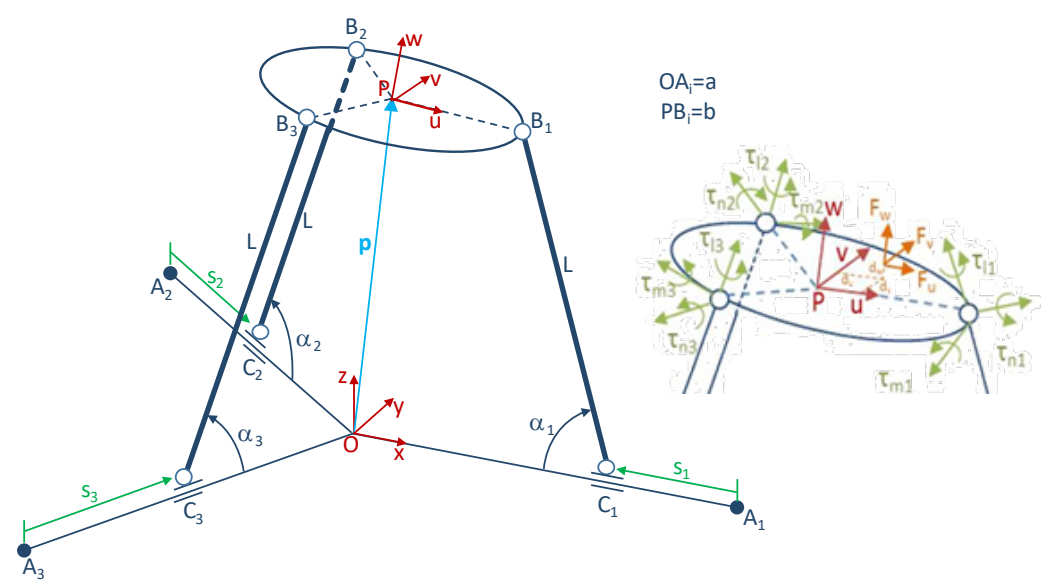

Fig. 2 Schematic of the compliant 3PRS manipulator and loads acting on the platform

For the study of the dynamics, all the elements will be considered as rigid bodies, whereas the joints will be treated as conventional using torsional springs to model their stiffness. That is, a pseudo-rigid model will be considered.

\subsection{Actuated plates}

The base of each leg performs a translation measured by $s_{i}$ under the force imposed by the actuators $F_{i}$ where $\mathrm{i}=1,2,3$, see Fig.2. Applying the Lagrange equations, the following equation of motion can be obtained, where $\mathbf{M}_{\text {act }}$ is the inertial matrix containing the mass of the plates.

$$
\mathbf{M}_{\mathbf{a c t}} \ddot{\mathbf{q}}=\mathbf{f}_{\mathbf{a c t}} \Rightarrow \mathbf{M}_{\text {act }}\left\{\begin{array}{lll}
\ddot{s}_{1} & \ddot{s}_{2} & \ddot{s}_{3}
\end{array}\right\}^{T}=\left\{\begin{array}{lll}
F_{1} & F_{2} & F_{3}
\end{array}\right\}^{T}
$$

\subsection{Platform translational dynamics}

To solve the dynamic problem of the platform, the translational and rotational dynamics of the platform have been decoupled. The generalized coordinates are defined by the position of the platform center $\mathbf{p}_{\mathbf{t}}=\left\{\begin{array}{lll}x_{P} & y_{P} & z_{P}\end{array}\right\}^{T}$. Considering 
the gravity $\mathbf{g}$ and the cutting forces due to the milling process as a force $\mathbf{f}$ applied in a position defined by vector $\mathbf{d}$ in the moving frame UVW, see Fig. 3 , the equations of motion of the platform translational dynamics are:

$$
\mathbf{R f}=\mathbf{M} \ddot{\mathbf{p}}_{\mathbf{t}}+\mathbf{g}
$$

Where $\mathrm{R}$ is the rotation matrix that relates the moving frame of the platform UVW with the fixed $X Y Z$.

$$
\mathbf{R}=\mathbf{R}_{\mathbf{y}}(\theta) \mathbf{R}_{\mathbf{X}}(\psi) \mathbf{R}_{\mathbf{z}}(\phi)=\left[\begin{array}{ccc}
c \theta c \phi+s \psi s \theta s \phi & -c \theta s \phi+s \psi s \theta c \phi & c \psi s \theta \\
c \psi s \phi & c \psi c \phi & -s \psi \\
-s \theta c \phi+s \psi c \theta s \phi & s \theta s \phi+s \psi c \theta c \phi & c \psi c \theta
\end{array}\right]
$$

Projecting to the manipulator coordinates, the contribution to the global dynamics is:

$$
\mathbf{J}_{\text {plat }_{\mathbf{t}}}^{T} \mathbf{f}=\mathbf{J}_{\text {plat }_{\mathbf{t}}}^{T} \mathbf{M J} \mathbf{p}_{\text {plat }_{\mathbf{t}}} \ddot{\mathbf{q}}+\mathbf{J}_{\text {plat }_{\mathbf{t}}}^{T} \mathbf{M} \mathbf{J}_{\text {plat }_{\mathbf{t}}} \dot{\mathbf{q}}+\mathbf{J}_{\text {plat }_{\mathbf{t}}}^{T} \mathbf{g}
$$

Where $J_{\text {platt }}$ is the Jacobian that relates the platform center coordinates $\boldsymbol{p}_{\mathbf{t}}$ with the generalized coordinates $\mathbf{q}$. The Jacobian is obtained analyzing the kinematics of the 3PRS. Taking into account the three loop closure equations and the three restrictions due to the planar motion of the legs in the planes at 120ㅇ, the following relations can be written:

$$
\mathbf{O P}+\mathbf{P B}_{\mathbf{i}}=\mathbf{O A}_{\mathbf{i}}+s_{i} \cdot \mathbf{s}_{\mathbf{i o}}+L \cdot \mathbf{l}_{\mathbf{i o}} \quad \mathbf{O B}_{i} \cdot \mathbf{u}_{i}=\left(\mathbf{O P}+\mathbf{P B}_{i}\right) \cdot \mathbf{u}_{i}=0
$$

After derivation, the 3PRS generalized coordinates $\mathbf{q}$ are related to the platform center velocity and the platform angular speed $\omega$ components, regarded here as quasi-velocities, by means of $\dot{\mathbf{p}}^{\mathbf{T}}=\left\{\begin{array}{llllll}\dot{x}_{p} & \dot{y}_{p} & \dot{z}_{p} & \omega_{x} & \omega_{y} & \omega_{z}\end{array}\right\}=\left\{\begin{array}{ll}\dot{\mathbf{p}}_{\mathbf{t}}^{\mathbf{T}} & \boldsymbol{\omega}^{\mathbf{T}}\end{array}\right\}$.

$$
\left.\begin{array}{c}
\mathbf{l}_{\mathbf{i o}} \cdot \dot{\mathbf{p}}_{\mathbf{t}}+\left(\mathbf{P B}_{\mathbf{i}} \wedge \mathbf{l}_{\mathbf{i o}}\right) \cdot \boldsymbol{\omega}=\mathbf{s}_{\mathbf{i o}} \cdot \mathbf{l}_{\mathbf{i o}} \cdot \dot{s}_{i} \\
\mathbf{u}_{\mathbf{i}} \cdot \dot{\mathbf{p}}_{\mathbf{t}}+\left(\mathbf{P B}_{\mathbf{i}} \wedge \mathbf{u}_{\mathbf{i}}\right) \cdot \boldsymbol{\omega}=\mathbf{0}
\end{array}\right\} \Rightarrow\left\{\begin{array}{c}
\mathbf{J}_{\mathbf{p} 1 i} \dot{\mathbf{p}}=\mathbf{J}_{\mathbf{q} i \mathbf{q}} \dot{\mathbf{q}} \\
\mathbf{J}_{\mathbf{p} 2 \dot{\mathbf{p}}=\mathbf{0} \dot{\mathbf{q}}}
\end{array}\right.
$$

Finally, the Jacobian of the platform is obtained as in Eq. (9), and divided in its translational and rotational part. 


$$
\left[\begin{array}{c}
\mathbf{J}_{\mathbf{p} \mathbf{1}} \\
\mathbf{J}_{\mathbf{p} \mathbf{2}}
\end{array}\right] \dot{\mathbf{p}}=\mathbf{J}_{\mathbf{p}} \dot{\mathbf{p}}=\left[\begin{array}{c}
\mathbf{J}_{\mathbf{q} 1} \\
\mathbf{0}
\end{array}\right] \dot{\mathbf{q}}=\mathbf{J}_{\mathbf{q}} \dot{\mathbf{q}} \Rightarrow \dot{\mathbf{p}}=\mathbf{J}_{\mathbf{p}}^{\mathbf{- 1}} \mathbf{J}_{\mathbf{q}} \dot{\mathbf{q}}=\mathbf{J}_{\mathbf{p l a t}} \dot{\mathbf{q}}=\left[\begin{array}{c}
\mathbf{J}_{\mathbf{p l a t}_{\mathbf{t}}} \\
\mathbf{J}_{\mathbf{p l a t}_{\mathbf{r}}}
\end{array}\right] \dot{\mathbf{q}}
$$

\subsection{Platform rotational dynamics}

To study the rotational dynamics of the platform, the Boltzmann-Hamel equations will be used, as the quasi-velocities $\omega$ will be the generalized local coordinates that define the orientation change of the platform. The quasi-velocities ca be related to the Euler rotations è as:

$$
\boldsymbol{\omega}=\mathbf{D}^{\mathbf{T}} \dot{\mathbf{e}}=\left[\begin{array}{ccc}
c \varphi & s \theta s \varphi & 0 \\
-s \varphi & s \theta c \varphi & 0 \\
0 & c \varphi & 1
\end{array}\right]\left\{\begin{array}{c}
\dot{\theta} \\
\dot{\psi} \\
\dot{\varphi}
\end{array}\right\}
$$

Quasi-velocities are also related to the 3PRS generalized coordinates by means of the platform rotational Jacobian $J_{\text {platr }}$ as in Eq. (9), so it is possible to relate Euler rotations and the 3PRS generalized coordinates by means of $\mathrm{J}_{\mathrm{e}}$ Jacobian.

$$
\boldsymbol{\omega}=\mathbf{D}^{\mathbf{T}} \dot{\mathbf{e}}=\mathbf{J}_{\mathbf{p l a t}_{\mathbf{r}}} \dot{\mathbf{q}} \Rightarrow \dot{\mathbf{e}}=\left[\mathbf{D}^{\mathbf{T}}\right]^{-1} \mathbf{J}_{\mathbf{p l a t}_{\mathbf{r}}} \dot{\mathbf{q}}=\mathbf{J}_{\mathbf{e}} \dot{\mathbf{q}}
$$

Using the Boltzmann-Hamel equations, the contribution of the platform rotational dynamics to the global is:

$$
\mathbf{J}_{\text {plat }_{\mathbf{r}}}^{T} \mathbf{m}=\mathbf{J} \mathbf{e}\left[\mathbf{D}^{\mathbf{T}} \mathbf{I}_{\mathbf{p l a t}} \mathbf{D}^{\mathbf{T}}\right] \mathbf{J}_{\mathbf{e}} \ddot{\mathbf{q}}+\mathbf{C}_{\text {plat }}
$$

Where $\mathbf{I}_{\text {plat }}$ is the inertia tensor in the moving frame, $\mathbf{c}_{\text {plat }}$ is a term that depends on the centrifugal and Coriolis forces and $\mathrm{m}$ is the vector of the moments acting on the platform.

$$
\mathbf{c}_{\mathbf{p l a t}_{\mathbf{r}}}=\mathbf{J}_{\mathbf{e}}^{\mathbf{T}}\left[\left[\mathbf{D}^{\mathbf{T}} \mathbf{I}_{\mathbf{p l a t}} \mathbf{D}^{\mathbf{T}}\right] \dot{\mathbf{J}}_{\mathbf{e}}+\left[\mathbf{D}^{\mathbf{T}} \mathbf{I}_{\mathbf{p l a t}} \dot{\mathbf{D}}^{\mathbf{T}}\right] \mathbf{J}_{\mathbf{e}}+\left[2 \dot{\mathbf{D}}^{\mathbf{T}} \mathbf{I}_{\mathbf{p l a t}}-\left(\frac{\partial \dot{\mathbf{\omega}}}{\partial \dot{\mathbf{e}}}\right)^{T} \mathbf{I}_{\mathbf{p l a t}}\right] \mathbf{J}_{\mathbf{p l a t}} \dot{\mathbf{q}}_{\mathbf{q}}\right.
$$

Regarding the loads on the platform, see Fig.2, two sources have been considered, the moments due to the cutting forces and the moments due to the spherical joints deflection. Although their elastic deflection can be considered in the potential energy used of the Lagrangian formulation, instead, here they have been regarded as external moments acting on the platform. Hence, the moments vector 
is Eq. (14), where $\mathbf{d}$ is the position of the tool center point with respect to the platform center.

$$
\mathbf{m}=\mathbf{m}_{\text {cut }}+\mathbf{m}_{\text {joints }}=\mathbf{R}(\mathbf{d} \wedge \mathbf{f})+\mathbf{m}_{\text {joints }}
$$

To compute the torques due to the spherical joints deflections, two coordinate systems have been located in each spherical joint, the first, $\mathbf{S}_{\mathrm{i}}$, fixed to the platform and the second, $S_{i o}$, fixed to the leg, see Fig. 3 for the leg 1 . In the zero position, at $\alpha_{i}=450$, both systems are identical. Rotations around the $m$ - and $n$-axes make reference to the joint deflection and rotation around the l-axis represents torsional deformation. To obtain the rotations between the systems $\mathbf{S}_{\mathbf{i}}$ and $\mathbf{S}_{\mathbf{i} 0}$, the rotation matrix that relates them must be developed. To do that, the unit vectors of each system $\boldsymbol{S}_{\text {io }}$ regarding to the global system XYZ must be calculated. These systems belong to the different limbs of length $L$, with $\mathbf{I}_{\mathbf{i} 0}$ aligned with the leg and $\mathbf{m}_{\mathbf{i} 0}$ parallel to the revolute joint axis. As a result, the rotation matrici $\mathbf{R}_{\mathbf{i}}$ to change from $S_{i o}$ that is placed in each leg to the fixed frame $X Y Z$ can be expressed as:

$$
\mathbf{R}_{\mathbf{i} 0}=\left[\begin{array}{lll}
\mathbf{m}_{\mathbf{i 0}} & \mathbf{n}_{\mathbf{i} 0} & \mathbf{l}_{\mathbf{i} 0}
\end{array}\right] \quad i=1,2,3
$$

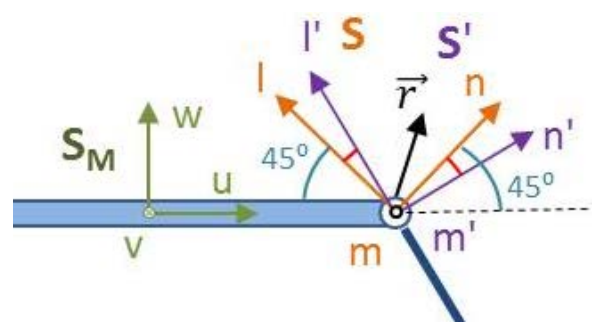

Fig. 3 Coordinate frames used to obtain the spherical joint rotations

On the other hand, the rotation matrices $\mathrm{Ri}$ that $\mathrm{Si}$ systems with $\mathrm{XYZ}$ are calculated. The unit vectors for each system Si can be defined by their fixed position in the platform, being $\alpha \mathrm{i} 0=45^{\circ}$ the default value of $\alpha \mathrm{i}$. To refer these vectors to the fixed frame, it is necessary to multiply them by the rotation matrix $\mathrm{R}$ that relates the moving frame attached to the platform UVW, with the fixed one XYZ:

$$
\mathbf{R}_{\mathbf{i}}=\left[\begin{array}{lll}
\mathbf{m}_{\mathbf{i}} & \mathbf{n}_{\mathbf{i}} & \mathbf{l}_{\mathbf{i}}
\end{array}\right]
$$

Finally, the rotation matrix $\mathbf{R}_{\mathbf{i}-\mathrm{io}}$ that relates frames $\mathbf{S}_{\mathbf{i o}}$ and $\mathbf{S}_{\mathbf{i}}$ is:

$$
\mathbf{R}_{\mathbf{i}-\mathbf{i 0}}=\mathbf{R}_{\mathbf{i 0}}^{T} \mathbf{R}_{\mathbf{i}} \quad i=1,2,3
$$


Furthermore, this matrix can be developed as a function of three rotations around all the axes system $S_{i o},\left(\beta_{m i}, \beta_{n i}, \beta_{l i}\right)$, where $\beta_{m i}$ and $\beta_{n i}$ are the two rotations due to the deflection of the joints, and $\beta_{\text {li }}$ is due to the torsion of the joints. Therefore, the rotation matrix that relates the systems $\mathbf{S}_{i}$ with $\mathbf{S}_{\mathbf{i} 0}$ may also be expressed as the product of the above rotation matrices.

$\mathbf{R}_{\mathbf{i}-\mathbf{i} \mathbf{0}}=\mathbf{R}_{\mathbf{m}}\left(\beta_{m}\right) \mathbf{R}_{\mathbf{n}}\left(\beta_{n}\right) \mathbf{R}_{\mathbf{l}}\left(\beta_{l}\right)=\left[\begin{array}{ccc}\mathrm{c} \beta_{n} \mathrm{c} \beta_{l} & -\mathrm{c} \beta_{n} \mathrm{~s} \beta_{l} & \mathrm{~s} \beta_{n} \\ \mathrm{~s} \beta_{m} \mathrm{~s} \beta_{n} \mathrm{c} \beta_{l}+\mathrm{c} \beta_{m} \mathrm{~s} \beta_{l} & -\mathrm{s} \beta_{m} \mathrm{~s} \beta_{n} \mathrm{~s} \beta_{l}+\mathrm{c} \beta_{m} \mathrm{c} \beta_{l} & -\mathrm{s} \beta_{m} \mathrm{c} \beta_{n} \\ -\mathrm{c} \beta_{m} \mathrm{~s} \beta_{n} \mathrm{c} \beta_{l}+\mathrm{s} \beta_{m} \mathrm{~s} \beta_{l} & \mathrm{c} \beta_{m} \mathrm{~s} \beta_{n} \mathrm{~s} \beta_{l}+\mathrm{s} \beta_{m} \mathrm{c} \beta_{l} & \mathrm{c} \beta_{m} \mathrm{c} \beta_{n}\end{array}\right]$ (18)

Equaling the terms of the matrices in Eq. (17) and (18), the rotations in the spherical joints can be obtained. The resulting expressions are:

$$
\beta_{m i}=\operatorname{atan}\left(\frac{-\mathbf{R}_{\mathbf{i}-\mathbf{i} \mathbf{0}(2,3)}}{\left.\mathbf{R}_{\mathbf{i}-\mathbf{i} \mathbf{0}(3,3)}\right)} \quad \beta_{n i}=\operatorname{asin}\left(\mathbf{R}_{\mathbf{i}-\mathbf{i} \mathbf{0}}(1,3)\right) \quad \beta_{l i}=\operatorname{atan}\left(\frac{-\mathbf{R}_{\mathbf{i}-\mathbf{i} \mathbf{0}}(1,2)}{\left.\mathbf{R}_{\mathbf{i}-\mathbf{i} \mathbf{0}(1,1)}\right)}\right.\right.
$$

Knowing the rotations produced in the spherical joints, the torques in each hinge can be obtained multiplying by the corresponding stiffness.

$$
\mathbf{m}_{\text {joints }}=\sum_{i=1}^{3}\left\{\boldsymbol{\tau}_{\mathbf{m i}}+\boldsymbol{\tau}_{\mathbf{n i}}+\boldsymbol{\tau}_{\mathbf{l i}}\right\}\left\{\begin{array}{l}
\tau_{m i}=k_{\mathrm{fsph}} \cdot \beta_{m i} \\
\tau_{n i}=k_{\mathrm{fsph}} \cdot \beta_{n i} \\
\tau_{l i}=k_{\mathrm{tsph}} \cdot \beta_{l i}
\end{array}\right.
$$

\subsection{Legs}

The position of the mass centers and the angular position of each limb have been considered as local generalized coordinates, $\mathbf{p}_{\mathbf{l e g i}}=\left\{\begin{array}{llll}x_{G i} & y_{G i} & z_{G i} & \alpha_{i}\end{array}\right\}$ , see Fig. 4. After obtaining the equations of motion of each leg, their contribution to the global dynamics of the manipulator is:

$$
\mathbf{J}_{\text {legi }}^{\mathbf{T}} \mathbf{M}_{\text {leg }} \mathbf{J}_{\text {legi }} \ddot{\mathbf{q}}+\mathbf{J}_{\text {legi }}^{\mathbf{T}} \mathbf{M}_{\text {leg }} \dot{\mathbf{J}}_{\text {legi }} \dot{\mathbf{q}}+\mathbf{J}_{\text {legi }}^{\mathbf{T}} \mathbf{k}_{\text {legi }}=\mathbf{0} \quad i=1,2,3
$$

Where $\mathbf{M}_{\mathbf{l e g}}$ is the mass matrix of each leg, $\mathbf{J}_{\text {legi }}$ are the Jacobians that relate $\mathbf{p}_{\text {legi }}$ with the 3PRS generalized coordinates $\mathbf{q}$, and $\mathbf{g} \mathbf{k}_{\text {legi }}$ is the vector that contains gravitational and elastic terms from the revolute joints.

Developing the closure loop equation for each leg and deriving, it is possible to relate the legs mass center position with the 3PRS generalized coordinates $\mathbf{q}$. 


$$
\frac{d}{d t}\left(\mathbf{O G}_{\mathbf{i}}=\mathbf{O A}_{i}+s_{i} \cdot \mathbf{s}_{\mathbf{i} \mathbf{0}}+\frac{L}{2} \cdot \mathbf{l}_{\mathbf{i} \mathbf{0}}\right) \Rightarrow \mathbf{J}_{G i} \dot{\mathbf{p}}_{\text {legi }}=\mathbf{J}_{q G i} \dot{\mathbf{q}} \quad i=1,2,3
$$

Also, to relate the legs angular position $\alpha_{i}$ with $\mathbf{q}$, it is necessary to average the three loop closure equations of the manipulator and derive:

$$
\frac{d}{d t}\left(\mathbf{O P}=\frac{1}{3} \sum_{i=1}^{3} \mathbf{O} \mathbf{A}_{\mathbf{i}}+s_{i} \mathbf{s}_{\mathbf{i} \mathbf{0}}+L \mathbf{l}_{\mathbf{i 0}}-\mathbf{P B} \mathbf{i}\right) \Rightarrow \quad \mathbf{J}_{\alpha i} \dot{\alpha}_{i}=\mathbf{J}_{q \alpha i} \dot{\mathbf{q}}
$$

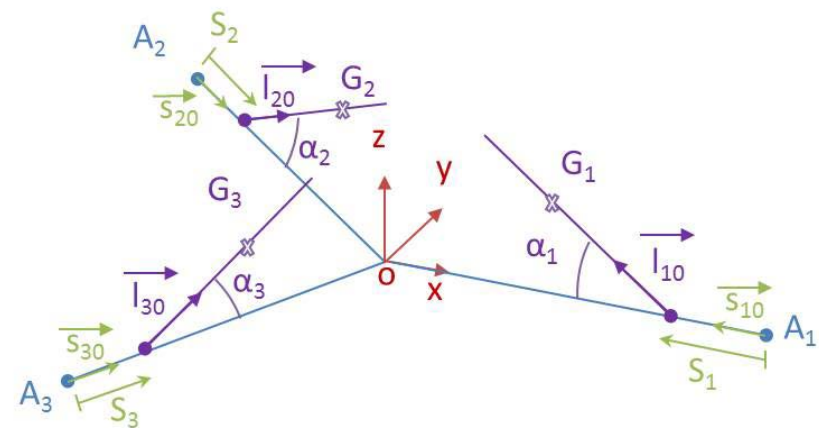

Fig. 4 Schematic representation of the legs variables

Finally, combining Eqs. (22) and (23), it is possible to obtain the three legs Jacobians.

$$
\left[\begin{array}{c}
\mathbf{J}_{G i} \\
\mathbf{J}_{\alpha i}
\end{array}\right] \dot{\mathbf{p}}_{\text {legi }}=\left[\begin{array}{c}
\mathbf{J}_{q G i} \\
\mathbf{J}_{q \alpha i}
\end{array}\right] \dot{\mathbf{q}} \Rightarrow \dot{\mathbf{p}}_{\text {legi }}=\mathbf{J}_{\text {legi }} \dot{\mathbf{q}}
$$

\subsection{Dynamic model of the manipulator}

Once the dynamic analyses for each of the components of the mechanism have been developed, it is necessary to group all of them in a single expression to model the whole manipulator. To do that, a summation of Eq. (3), (6), (12) and (21) is performed. The resultant expression is:

$$
\begin{aligned}
& {\left[\mathbf{M}_{\text {act }} \ddot{\mathbf{q}}\right]+\left[-\mathbf{J}_{\text {plat }_{\mathbf{t}}}^{T} \mathbf{f}+\mathbf{J}_{\text {plat }_{\mathbf{t}}}^{T} \mathbf{M J}_{\text {plat }_{\mathbf{t}}} \ddot{\mathbf{q}}+\mathbf{J}_{\text {plat }_{\mathbf{t}}}^{T} \mathbf{M} \mathbf{J}_{\text {plat }_{\mathbf{t}}} \dot{\mathbf{q}}+\mathbf{J}_{\text {plat }_{\mathbf{t}}}^{T} \mathbf{g}\right]+} \\
& +\left[-\mathbf{J}_{\mathbf{p l a t}_{\mathbf{r}}}^{T} \mathbf{m}+\mathbf{J}_{\mathbf{e}}^{\mathbf{T}}\left[\mathbf{D}^{\mathbf{T}} \mathbf{I}_{\mathbf{p l a t}} \mathbf{D}^{\mathbf{T}}\right] \mathbf{J}_{\mathbf{e}} \ddot{\mathbf{q}}+\mathbf{c}_{\mathbf{p l a t}_{\mathbf{r}}}\right]+ \\
& +\sum_{i=1}^{3}\left[\mathbf{J}_{\operatorname{leg} i}^{\mathbf{T}} \mathbf{M}_{\mathbf{l e g}} \mathbf{J}_{\mathbf{l e g} i} \ddot{\mathbf{q}}+\mathbf{J}_{\operatorname{leg} i}^{\mathbf{T}} \mathbf{M}_{\mathbf{l e g}} \dot{\mathbf{J}}_{\mathbf{l e g} i} \dot{\mathbf{q}}+\mathbf{J}_{\operatorname{leg} i}^{\mathbf{T}} \mathbf{g k} \mathbf{k}_{\mathbf{l e g} i}\right]=\mathbf{f}_{\mathbf{a c t}}
\end{aligned}
$$




\subsection{Joints stiffness}

To design and analyze the performance of 3PRS compliant parallel mechanism joints, ANSYS Workbench FEM software has been used. The mesh applied to the mechanism consists of a quadratic tetrahedral mesh. The nodes size in the flexure joints has been reduced to obtain more accurate results in the areas with a high deformation and stress concentration. After checking the stresses and displacements reached for several joint dimensions, the dimensions of the revolute joints are $8 \mathrm{~mm}$ radius and $2 \mathrm{~mm}$ of minimum thickness, for the spherical joints, $13 \mathrm{~mm}$ length, $3 \mathrm{~mm}$ of minimum diameter and a fillet radius of $4 \mathrm{~mm}$. The stiffness if the joints have been also measured. For the revolute hinge, the achieved value has been $k_{\text {rev }}=98.37 \mathrm{Nm} / \mathrm{rad}$. For the spherical joint, the bending stiffness and the torsional stiffness have been $k_{\mathrm{fsph}}=32.67 \mathrm{Nm} / \mathrm{rad}$ and $k_{\mathrm{tsph}}=24.46 \mathrm{Nm} / \mathrm{rad}$.

\section{Experimental validation}

An initial prototype in aluminum $7075 T 6$ has been built, see Fig. 5 . The three actuators are based on a linear belt drive Igus ZLW-1040-02-S-100 coupled to a DC RE40 Maxon servomotor with a GP-32 14:1 reduction. A NI-PXle 1062 has been used to control the prototype motion in real time with a cascaded joint space control of position, velocity and current. The position loop cycle time is $5 \mathrm{~ms}$.

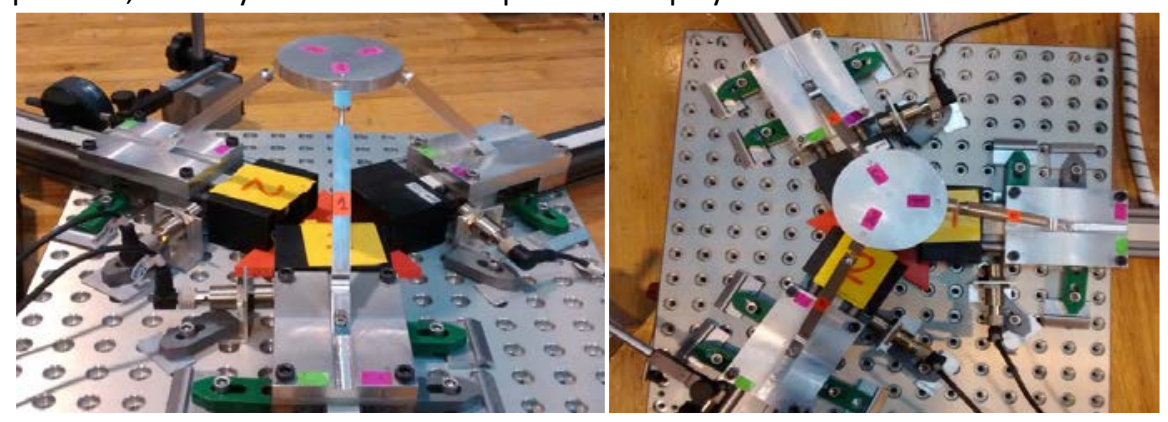

Fig. 5 Developed prototype

Currently, intensive testing is being made to validate the results from the $d y$ namic model. The main sources of uncertainty are the friction in the linear guides, the FEA estimated stiffness, the simplified kinematics of the joints, the bolted joints not modelled in FEA and the manufacturing and assembly errors on the prototype. Nevertheless, in Figs. 6 and 7 it can be seen the torque measured at the motors, which is an indirect measurement of the force, for two motions, first a sinusoidal motion in $\mathrm{Z}$ with an amplitude of $2 \mathrm{~mm}$ and $0,5 \mathrm{~Hz}$, and then a sinusoidal motion of the three actuators with the same amplitude and frequency but a phase 
shift of 120 . Although the results are quite similar, there are deviations and even the trend is different, in the first test the simulated torque is lower and vice versa, so further testing must be made to adjust the model parameters to the real ones.
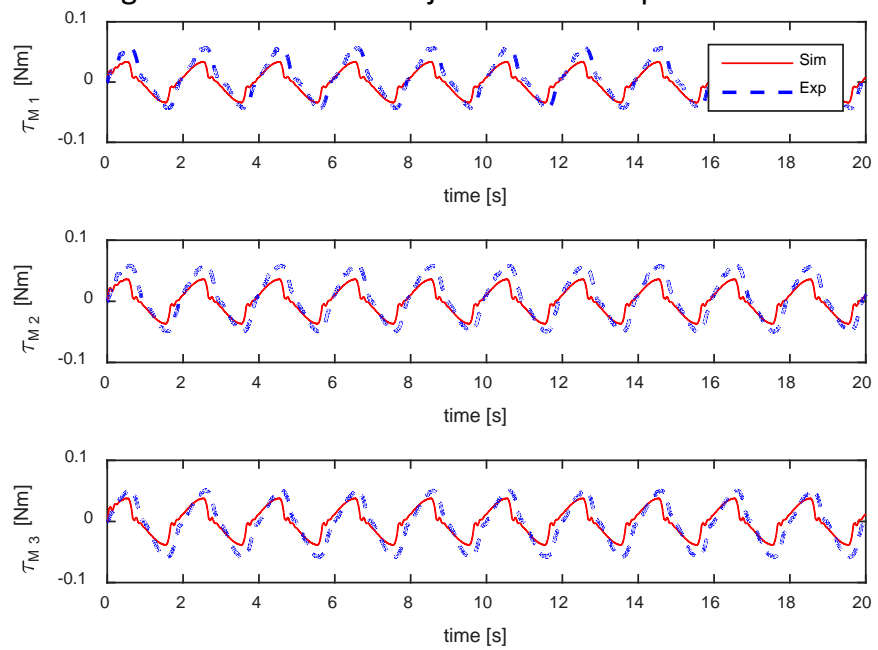

Fig. 6 Simulated motor torque (red continuous line) vs. measured (blue discontinuous line)
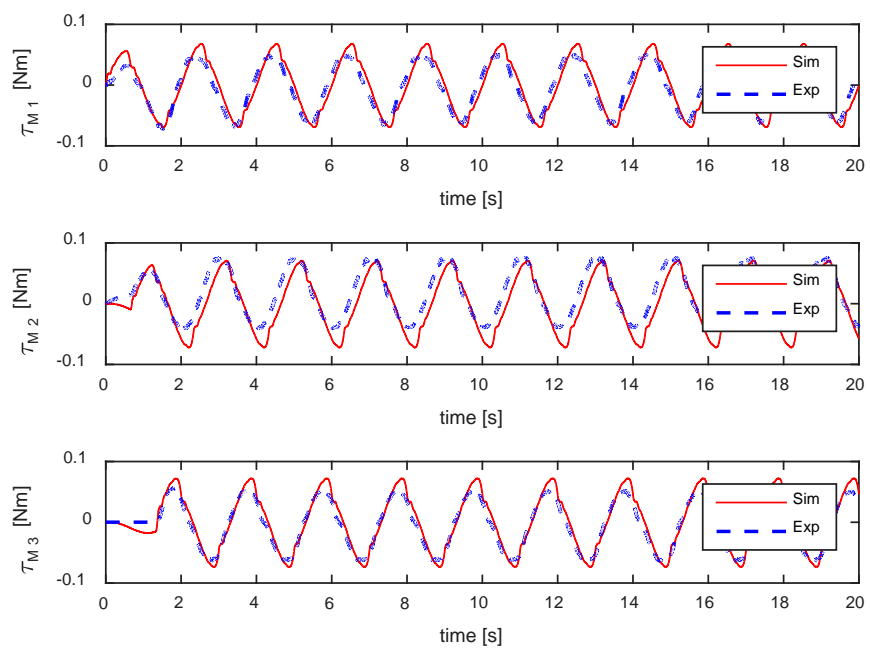

Fig. 7 Simulated motor torque (red continuous line) vs. measured (blue discontinuous line) 


\section{Conclusions}

The present work shows a procedure to model the dynamics of a compliant 3PRS parallel manipulator. Given the fact that the compliant joints are relatively small in size comparing to the limbs and platform dimensions, it has been assumed that all the bodies behave as rigid, and the joints have been modelled as lumped torsional springs. The method used is the Principle of Energy Equivalence, combined with the Bolttzmann-Hamel equations to study the rotational dynamics of the platform. A prototype has been constructed for validation. Although there are several sources of discrepancy that are being analyzed, the initial results seem to be promising.

Acknowledgments The authors of this paper wish to acknowledge the financial support received from the Spanish Government via the Ministerio de Economía y Competitividad (Project DPI201564450-R), the ERDF of the European Union, the Government of the Basque Country (Project GICO7/78, IT445-10 and SAIOTEK 2013 SAI13/245, SPC13UNO11), and the University of the Basque Country (Zabalduz-2012).

\section{References}

Merlet J P (2000) Parallel Robots, Kluwer Academic Publishers, London.

Howell L (2001) Compliant Mechanisms, Wiley, New York.

Choi Y, Kim J (2012) A millimeter-range flexure-based nano-positioning stage using a self-guided displacement amplification mechanism. Mech Mach Theory 50: 109-120

Kenton B, Leang K (2012) Design and Control of a Three-Axis Serial-Kinematic High-Bandwidth Nanopositioner. IEEE/ASME Transactions on Mechatronics, vol $17 \mathrm{~N} 2$

Hao G, Kong X (2012) Design and Modeling of a Large-Range Modular XYZ Compliant Parallel Manipulator Using Identical Spatial Modules. Journal of Mechanisms and Robotics, Vol. 4 / 021009

Li Y, Xu Q (2007) Kinematic analysis of a 3-PRS parallel manipulator. Robotics and ComputerIntegrated Manufacturing 23: 395-408

Altuzarra O, Eggers P, Campa FJ, Roldan-Paraponiaris C, Pinto C (2015) Dynamic modelling of lower-mobility parallel manipulators using the Boltzmann-Hamel equations, $3^{\text {rd }}$ Conference on Mechanisms, Transmissions and Applications (MeTrApp 2015), 6-8 May

Choi H, Han Ch, Wang W (2011) 2-DOF Kinematic XY stage design based on flexure element. Proceeding of the IEEE International Conference on Mechatronics and Automation. Beijing, China, 1412-1417 
Aphale S, Moheimani S, Yong Y (2009) Design, Identification, and Control of a Flexure-Based XY Stage for Fast Nanoscale Positioning. Of 10th IEEE Transactions on Nanotechnology, vol. 8, no. $1,46-54$

Ahn D, Chun B, Gweon D, Kim H (2010) Development and optimization of a novel 3-DOF precision flexure stage. Proceeding of the IEEE International Conference on Nanotechnology Joint Symposium with Nano Korea. Kintex, Korea, 903-906

Gao F, Ge Q, Yue Y, Zhao X (2010) Relationship among input-force, payload, stiffness and displacement of a 3-DOF perpendicular parallel micro-manipulator. Mech Mach Theory 45; 756-771 